\title{
Wieso ich profitiere, wenn Du länger Arbeitslosengeld erhältst
}

\author{
Valentina Sontheim
}

\section{Relevanz}

Wie hoch soll das Arbeitslosengeld ausfallen und wie lange soll es ausbezahlt werden? Die Arbeitslosenversicherung (ALV) führt dazu, dass Arbeitslose weniger intensiv nach Stellen suchen und somit länger arbeitslos bleiben. Damit steht die Politik vor dem Dilemma, dass sie mit der ALV die Arbeitenden vor Einkommensverlusten schützen will, aber dadurch gleichzeitig die Motivation beeinträchtigt, einen Job zu suchen. Das Politikdilemma schwächt sich etwas ab, wenn in wirtschaftlich schwierigen Zeiten eine Verlängerung der Arbeitslosenunterstützung den Arbeitsmarkt entlastet und zu positiven Nebeneffekten für die verbleibenden Jobsuchenden führt. Die ALV kann ein Instrument sein, die vorübergehenden Härten eines besonderen Strukturwandels abzufedern.

Beatrix Eugster

\section{Quelle}

Der nachfolgende Text ist eine Zusammenfassung von: Rafael, Lalive, Camille Landais und Josef Zweimüller (2015), Market Externalities of Large Unemployment Insurance Extension Programs, American Economic Review 105(12), 3564-96.

Einen passenden Job zu finden ist bei weitem nicht immer einfach. Wenn die freien Stellen knapp sind und die Konkurrenz auf dem Arbeitsmarkt groß ist, sind

\author{
V. Sontheim $(\bowtie)$ \\ Universität St. Gallen, St. Gallen, Schweiz \\ E-Mail: valentina.sontheim@student.unisg.ch \\ (C) Der/die Autor(en) 2018 \\ C. Keuschnigg (Hrsg.), Inklusives Wachstum und wirtschaftliche Sicherheit, \\ https://doi.org/10.1007/978-3-658-21344-2_9
}


die Aussichten auf einen schnellen Erfolg bei der Jobsuche gering. Die Arbeitslosen können jedoch selbst die Erfolgswahrscheinlichkeit erhöhen, indem sie viele Bewerbungen aussenden, häufig Interviews machen, und damit intensiver nach einem passenden Job suchen. Die Arbeitslosenversicherung (ALV) mindert jedoch den Anreiz zur Jobsuche, verlängert die Dauer der Arbeitslosigkeit und trägt damit selbst zu höherer Arbeitslosigkeit bei. Wird die ALV ausgeweitet, dann bleibt das Einkommen auch in der Arbeitslosigkeit hoch. Umso weniger können die Arbeitslosen gewinnen, wenn sie eine neue Beschäftigung aufnehmen. Die finanziellen Anreize zur Jobsuche nehmen ab. Eine Ausweitung der ALV beeinflusst aber nicht nur die Verhaltensweise der Arbeitnehmer, sondern auch die Bedingungen auf dem Arbeitsmarkt. Wenn die Arbeitslosen weniger intensiv nach Arbeit suchen und daher weniger Leute um gleich viele offene Stellen konkurrieren, dann wird es für die verbleibenden Arbeitssuchenden leichter, eine passende Stelle zu bekommen. Wenn in wirtschaftlich schwierigen Zeiten oder bei einem ausgeprägten Strukturwandel besonders betroffene Gruppen länger Arbeitslosengeld erhalten, profitieren die übrigen Arbeitssuchenden. Das kann besondere Härten eines starken Strukturwandels abfedern.

Die Schwierigkeiten, einen Job zu finden, hängen jedoch nicht nur von den Bemühungen der Arbeitssuchenden, sondern auch von der Anzahl offener Stellen ab. Eine großzügige ALV mindert tendenziell die Arbeitsnachfrage der Unternehmen. Wenn die Arbeitslosenversicherung großzügiger wird, müssen nämlich auch die Löhne steigen, um die finanziellen Anreize für die Jobsuche und Annahme einer Beschäftigung zu erhalten. Für die Firmen wird es mit höheren Löhnen teurer, neue Stellen zu schaffen. Wenn in der Folge die Anzahl offener Stellen zurückgeht, wird eine erfolgreiche Jobsuche schwieriger. Bei gleicher Anstrengung der Arbeitssuche sinkt die Wahrscheinlichkeit, einen Job zu finden. In der Praxis zeigt sich jedoch, dass die Löhne nicht bemerkenswert steigen, wenn die Dauer der gewährten Arbeitslosenunterstützung verlängert wird. Die Bezahlung für die meisten Jobs ist ausreichend hoch im Vergleich zur gewährten Arbeitslosenentschädigung. Wenn die Löhne nicht oder nur leicht steigen, kann eine längere Dauer der ALV für besonders betroffene Gruppen die Arbeitsmarktchancen der verbleibenden Arbeitssuchenden verbessern.

Lalive, Landais und Zweimüller untersuchen empirisch, wie sich eine Verlängerung der Bezugsdauer der ALV auswirkt, wenn nur eine spezielle Gruppe von Arbeitnehmern Anspruch darauf hat, aber andere nicht. Konkret analysieren die Forscher ein regionales Programm der ALV in Österreich, welches die Arbeitsmarktfolgen eines regional konzentrierten wirtschaftlichen Einbruchs für besonders benachteiligte Gruppen lindern sollte und von 1988 bis 1993 in 28 von ca. 100 Bezirken die maximale Bezugsdauer von Taggeldern um drei Jahre angehoben hat. 
Diese Veränderung ist angesichts der bis anhin geltenden maximalen Bezugsdauer von 52 Wochen (Arbeitslose über 50) bzw. 39 Wochen (Arbeitslose zwischen 40 und 49) erheblich. Von der Verlängerung profitierten allerdings nur ältere Arbeiter mit durchgehender Beschäftigung während der letzten 25 Jahre. Keinen Anspruch hatten dagegen jüngere Arbeitslose und solche mit zeitweiligen Arbeitsunterbrüchen in der Vergangenheit. Wie ändern sich die Anreize zur Jobsuche und welche Folgen hat das Programm auf die Bedingungen auf dem Arbeitsmarkt? Wie reagieren die begünstigten Arbeitslosen und wie die anderen Arbeitssuchenden, die nicht direkt von der Verlängerung der Bezugsdauer profitieren und daher die Auswirkungen nur indirekt zu spüren bekommen?

\section{Von 1988 bis 1993 wurde in Österreich zur Abfederung eines regional konzentrierten Strukturwandels in 28 von ca. 100 Bezirken die maxi- male Bezugsdauer des Arbeitslosengeldes für ältere Arbeitnehmer mit langer Beschäftigung um drei Jahre angehoben.}

Um die Folgen der Reform für den Arbeitsmarkt zu ermitteln, untersuchen die Wissenschaftler die Auswirkungen auf zwei Gruppen von Arbeitssuchenden, die sich auf dem gleichen regionalen Arbeitsmarkt um dieselben Stellen bewerben. Konkret vergleichen sie Arbeitslose mit Wohnort in Bezirken, welche die Reform umsetzen, mit solchen in anderen Bezirken, in welchen die ALV unverändert bleibt. Dabei interessiert besonders der indirekte Effekt der Reform, der sich aus der Stellenkonkurrenz auf dem Arbeitsmarkt ergibt. Wie verändert das Programm bei unveränderter Anstrengung in der Jobsuche die Chancen auf einen Job? Die Forscher wollen vor allem wissen, wie Arbeitslose betroffen sind, welche die Kriterien für die Zulassung zum Programm nicht erfüllen. Sie haben keinen direkten Nutzen von der Reform, da sie nicht von einer Verlängerung der Bezugsdauer profitieren. Wenn jedoch die begünstigten Arbeitnehmer weniger stark suchen und länger vom Arbeitsmarkt fernbleiben, dann konkurrieren weniger Arbeitslose um die Zahl freier Stellen. Damit haben es auch die nicht geförderten Jobsuchenden leichter, weil die geringere Konkurrenz auf dem Stellenmarkt ihre Erfolgsaussichten auf einen Job verbessert.

Das Programm hat beachtliche Auswirkungen auf beide Gruppen von Arbeitslosen. Jene, welche die Unterstützung nun wesentlich länger beziehen können, nehmen sich in ihren Anstrengungen zur Stellensuche deutlich zurück. Bei ihnen steigt in der Folge die Arbeitslosendauer im Durchschnitt um 43 Wochen an, gegenüber einer durchschnittlichen Dauer der Arbeitslosigkeit von 21 Wochen vor Einführung des Programms (Abb. 1, Grafik A). Dieser Effekt hält nur solange an, wie auch die Reform in Kraft ist, und verschwindet danach wieder. 


\section{Effekt des REBP-Programms auf die Arbeitslosendauer}

A. Direkt betroffene Arbeitslose

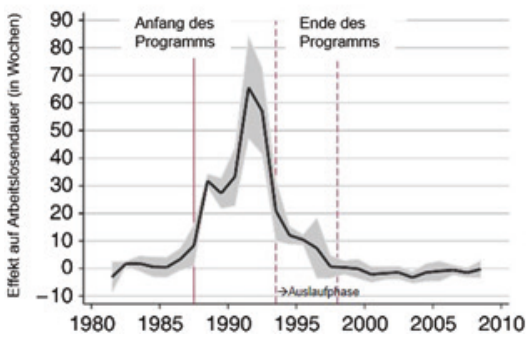

B. Nicht direkt betroffene Arbeitslose

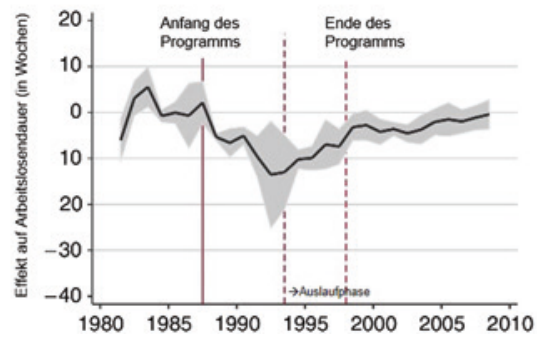

Abb. 1 Effekt des REBP-Programms auf die Arbeitslosendauer. (Quelle: Rafael u. a., 2015, 3581)

Die Wissenschaftler stellen zudem fest, dass auch die nicht begünstigten Jobsuchenden markant profitieren, weil der Rückzug der geförderten Arbeitslosen die Stellenkonkurrenz auf dem Arbeitsmarkt entschärft. Sie müssen im Durchschnitt 4 Wochen weniger lang suchen, bis sie einen passenden Job finden, das entspricht einer um $20 \%$ geringeren Arbeitslosendauer (Abb. 1, Grafik B).

Mit Einführung des Programms steigt bei den geförderten Arbeitnehmern die Arbeitslosendauer um 43 Wochen an. Aufgrund geringerer Stellenkonkurrenz verkürzt sich die Arbeitslosigkeit bei den übrigen Stellensuchenden um 4 Wochen.

Das Programm begünstigt nur ältere Arbeitnehmer mit langer Beschäftigungsdauer und hat daher recht unterschiedliche Auswirkungen auf verschiedene Altersgruppen. Arbeitslose über 50 Jahre, die nicht zum Programm zugelassen sind, konkurrieren am ehesten mit der begünstigten Gruppe. Deshalb ist es naheliegend, dass sie die Folgen der Reform am stärksten zu spüren bekommen. Sie können am ehesten von einer höheren Wahrscheinlichkeit einen Job zu finden profitieren. Von allen Arbeitslosen, die keinen Anspruch auf eine längere Bezugsdauer des Arbeitslosengeldes haben, sind nach Start des Programms die 46 bis 49-Jährigen um durchschnittlich 2-3 Wochen weniger lang arbeitslos. Bei den über 50-Jährigen ist der Effekt mit 6-9 Wochen wesentlich stärker.

Zusätzlich stellen Lalive, Landais und Zweimüller fest, dass sich das Programm auch positiv auf benachbarte Bezirke auswirkt. Stellensuchende in anderen Bezirken, deren Arbeitsmärkte stark mit den Reform-Bezirken vernetzt sind, profitieren 
ebenfalls. Ihre durchschnittliche Arbeitslosendauer verkürzt sich um 4 Wochen, da mit Einführung des Programms die Konkurrenz der Stellensuchenden aus den Nachbarsbezirken nachlässt.

Das Programm ist auf benachteiligte Gruppen zugeschnitten, die in einzelnen Regionen von verschlechterten Wirtschaftsbedingungen besonders stark betroffen sind. Die Wissenschaftler ziehen den Schluss, dass eine temporäre Verlängerung der Bezugsdauer für besonders betroffene Gruppen weniger Kosten mit sich bringt als bisher erwartet, da ein solches Programm auch positive Auswirkungen auf andere Stellensuchende hat.

Open Access Dieses Kapitel wird unter der Creative Commons Namensnennung 4.0 International Lizenz (http://creativecommons.org/licenses/by/4.0/deed.de) veröffentlicht, welche die Nutzung, Vervielfältigung, Bearbeitung, Verbreitung und Wiedergabe in jeglichem Medium und Format erlaubt, sofern Sie den/die ursprünglichen Autor(en) und die Quelle ordnungsgemäß nennen, einen Link zur Creative Commons Lizenz beifügen und angeben, ob Änderungen vorgenommen wurden.

Die in diesem Kapitel enthaltenen Bilder und sonstiges Drittmaterial unterliegen ebenfalls der genannten Creative Commons Lizenz, sofern sich aus der Abbildungslegende nichts anderes ergibt. Sofern das betreffende Material nicht unter der genannten Creative Commons Lizenz steht und die betreffende Handlung nicht nach gesetzlichen Vorschriften erlaubt ist, ist für die oben aufgeführten Weiterverwendungen des Materials die Einwilligung des jeweiligen Rechteinhabers einzuholen.

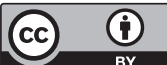

